
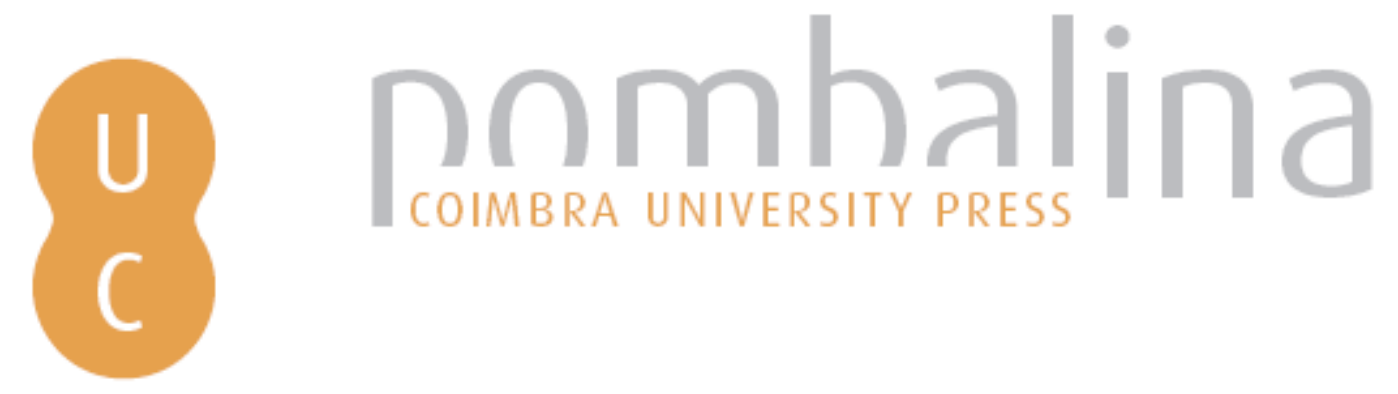

\title{
AgIM: ensino pós-graduado em gestão de informação agrícola e agricultura de recisão em Cabo Verde e Moçambique
}

Autor(es): $\quad$ Painho, Marco; Baptista, Alexandre; Nascimento, Judite; Atumane, Ali

Publicado por: Imprensa da Universidade de Coimbra

URL

persistente: URI:http://hdl.handle.net/10316.2/37103

DOI: DOI:http://dx.doi.org/10.14195/978-989-26-0983-6_50

Accessed : $\quad$ 26-Apr-2023 04:23:04

A navegação consulta e descarregamento dos títulos inseridos nas Bibliotecas Digitais UC Digitalis, UC Pombalina e UC Impactum, pressupõem a aceitação plena e sem reservas dos Termos e Condições de Uso destas Bibliotecas Digitais, disponíveis em https://digitalis.uc.pt/pt-pt/termos.

Conforme exposto nos referidos Termos e Condições de Uso, o descarregamento de títulos de acesso restrito requer uma licença válida de autorização devendo o utilizador aceder ao(s) documento(s) a partir de um endereço de IP da instituição detentora da supramencionada licença.

Ao utilizador é apenas permitido o descarregamento para uso pessoal, pelo que o emprego do(s) título(s) descarregado(s) para outro fim, designadamente comercial, carece de autorização do respetivo autor ou editor da obra.

Na medida em que todas as obras da UC Digitalis se encontram protegidas pelo Código do Direito de Autor e Direitos Conexos e demais legislação aplicável, toda a cópia, parcial ou total, deste documento, nos casos em que é legalmente admitida, deverá conter ou fazer-se acompanhar por este aviso.

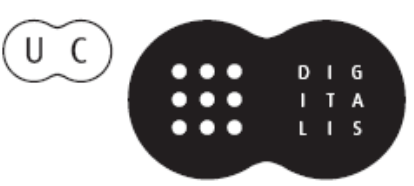




\section{$\forall$ \\ TAS DAS I JORNADAS LUSÓFONAS DE CIÊNCIAS E TECNOLOGIAS DE INFORMAÇÃO GEOGRÁFICA}

Editores

José Gomes dos Santos

Cidália Fonte

Rui Ferreira de Figueiredo

Alberto Cardoso

Gil Gonçalves

José Paulo Almeida

Sara Baptista 


\section{ARTIGO 50}

\section{AgIM - ENSINO Pós-gRAdUAdo EM GESTÃo DE INFORMAÇÃo Agrícola E AgRICULTURA DE PRECISÃO EM CABO VERDE E MOÇAMBIQUE}

PAINHO, Marco ${ }^{1}$; BAPTISTA, Alexandre ${ }^{2}$ NASCIMENTO, Judite 3 \& ATUMANE, Alí

\footnotetext{
${ }^{1}$ Instituto Superior de Estatística e Gestão de Informação - Universidade Nova de Lisboa (Portugal) Campus de Campolide, 1070-312 Lisboa, Portugal; Tel: +351 213 828616; Fax: +351 213 828611; email: painho@isegi.unl.pt

2 Instituto Superior de Estatística e Gestão de Informação - Universidade Nova de Lisboa (Portugal); Campus de Campolide, 1070-312 Lisboa, Portugal; Tel: +351 213 828610; Fax: +351 213 828611; email: a.baptista@isegi.unl.pt

3 Universidade de Cabo Verde (Cabo Verde); Praça António Lereno, 379C - Praia, Santiago, Cabo Verde; Tel: (00238) 26199 04; Fax: (00238) 26126 60; email: judite.nascimento@adm.unicv.edu.cv

${ }^{4}$ Faculdade de Agricultura - Universidade Católica de Moçambique (Moçambique); Rua do Aeroporto, 22 - Cuamba, Moçambique; Tel: +258 825 961720; email: aatumane@ucm.ac.mz
}

\section{RESUMO}

Apesar da recente expansão do ensino superior e a crescente procura de profissionais em Gestão de Informação Agrícola e Agricultura de Precisão, Moçambique e Cabo Verde ainda apresentam uma escassez de especialistas altamente qualificados, que tenham a capacidade de formar as futuras gerações de profissionais. Com o objetivo de dar uma resposta adequada à falta de mão-de-obra qualificada, num sector considerado estrategicamente relevante para o desenvolvimento socioeconómico dos dois países, o Instituto Superior de Estatística de Gestão de Informação da Universidade Nova de Lisboa (ISEGI-NOVA), em parceria com a Universidade de Cabo Verde (UniCV) e a Universidade Católica de Moçambique (UCM) e com o apoio da Esri Portugal, desenvolveu um projeto (AgIM - Agricultural Information 
Management and Precision Farming) com um período de execução de 42 meses. Pretende-se através da utilização dos SIG, a capacidade para resolução de problemas locais e regionais e condições para multiplicar os benefícios da transferência do know-how do curso de Gestão de Informação Agrícola e Agricultura de Precisão. A primeira edição do Mestrado e Pós-Graduação em Moçambique teve início em17 de março de 2014, estando previsto o início da $1^{\text {a }}$ edição em Cabo Verde em setembro de 2014.

\title{
PALAVRAS-CHAVE
}

Ensino pós-graduado, Sistemas de informação geográfica, Agricultura de precisão, Gestão de informação agrícola e Blended learning.

\section{AgIM - POST-graduAted EDUCATION IN AgRiculture INFORMATION MANAGEMENT AND PRECISION FARMING IN CAPE VERDE AND MOZAMBIQUE}

\begin{abstract}
Despite the recent expansion of higher education and the growing demand for professionals in Agricultural Information Management and Precision Farming, Mozambique and Cape Verde still have a shortage of highly qualified specialists who have the ability to train future generations of professionals. In order to give an adequate response to the lack of skilled labor in a sector considered strategically relevant to the socio-economic development of both countries, the Instituto Superior de Estatística de Gestão de Informação da Universidade Nova de Lisboa (ISEGI-NOVA) in partnership with the Universidade de Cabo Verde (UniCV) and the Universidade Católica de Moçambique (UCM) and with support from Esri Portugal, developed a project (AgIM - Agricultural Information Management and Precision Farming) with an implementation period of 42 months. It is intended by the use of GIS, the ability to solve local and regional problems and able to multiply the benefits of transferring the course know-how on Agricultural Information Management and Precision Farming. The first edition of the Master and Postgraduate Course in Mozambique began on March $17^{\text {th }}, 2014$ and in Cape Verde is scheduled to begin in September 2014.
\end{abstract}

\section{KEYWORDS}

Post-graduated teaching, Geographic information systems, Precision farming, Agriculture information management, Blended learning. 


\section{INTRODUÇÃO}

Nas duas últimas décadas tem ocorrido um interesse crescente pelo conceito de agricultura de precisão. Este ramo científico tem sido suportado pelos recentes avanços não só nas áreas tradicionais das ciências agrárias como também na emergência e convergência de novas metodologias de processamento nos campos dos Sistemas de informação geográfica (SIG), sistemas de posicionamento global (GPS) e deteção remota que contribuíram para o conceito de variabilidade espacial e temporal. Segundo ADHIKARI (2009) com o desenvolvimento do potencial destas tecnologias, levou a um novo conceito de agricultura focado nos fatores de produção com os seguintes objetivos:

1. Aumento da eficiência da produção e dos lucros

2. Aumento da qualidade de produção

3. Uso eficiente e sustentável de químicos e pesticidas

4. Eficiência energética

5. Proteção do solo e águas subterrâneas

Existem no entanto fatores que limitam a adoção e implementação da agricultura de precisão (KITCHEN et al, 2002; WIEBOLD et al, 1998), especialmente em países em vias de desenvolvimento, onde os custos e a falta de profissionais qualificados são evidentes. Tendo em conta os objetivos da agricultura de precisão: otimização de recursos e sustentabilidade ambiental, esta deveria ser vista como fundamental no desenvolvimento da atividade agrícola destes países, mesmo que a sua introdução e técnicas sejam diferentes do que é implementado na Europa ou América do Norte (COOK et al, 2003; LUMPKIN, 2011; MAOHUA, 2001; MONDAL \& BASU, 2009; RILWANI \& IKHUORIA, 2006). Neste contexto, foram propostas estratégias de adoção da agricultura de precisão em países em vias de desenvolvimento. MISHRA et al (2003) apresentou um plano focado na sua integração na Índia: Criação de equipas multidisciplinares para analisar o raio de ação da agricultura de precisão; Criação de cooperativas de agricultores para auxiliar na aquisição de ferramentas utilizadas na agricultura de precisão; Legislação restritiva do uso indiscriminado de produtos químicos e pesticidas causadores de problemas ecológicos/ ambientais; Realização de estudos-piloto junto dos agricultores para de- 
monstrar os benefícios da agricultura de precisão.

Em Cabo Verde e Moçambique não existe atualmente nenhuma instituição de ensino superior que ofereça um curso de agricultura de precisão. Tendo em conta a importância do setor agrícola para a economia dos dois países e uma escassez de profissionais qualificados, o ISEGI-NOVA desenvolveu uma parceria com a UniCV e a UCM, no sentido de criar nas duas instituições um curso de pós-graduação e mestrado em gestão de informação agrícola e agricultura de precisão.

\section{SITUAÇÃo DO ENSINO SUPERIOR E SETOR AGRÍCOLA EM MOÇAMBIQUE E CABO VERDE}

Desde a promulgação da Lei de Ensino Superior em 1993, Moçambique tem registado progressos significativos que culminaram em 2000 com a criação do Ministério do Ensino Superior, Ciência e Tecnologia que tem o papel de coordenar a expansão do sector e promover a equidade no acesso às IES. Com a implementação do Plano Estratégico para o Ensino Superior (PEES 2000-2010), registou-se um aumento do número de instituições públicas e privadas (o país conta atualmente com 38). No entanto a quantidade de alunos continua a ser uma das mais reduzidas da região e ainda permanecem problemas ao nível da qualidade das infraestruturas e do corpo docente. Existe também um desequilibro entre os cursos oferecidos e a sua relevância para o desenvolvimento de Moçambique, com a maioria dos estudantes a enveredar pelas ciências sociais em detrimento das ciências naturais e áreas tecnológicas.

Relativamente ao panorama socioeconómico de Moçambique, mais de $70 \%$ das famílias com baixos rendimentos habita em áreas rurais. A agricultura é o seu principal meio de subsistência, mas a sua produtividade é muito reduzida. Estas comunidades são extremamente vulneráveis a desastres naturais como secas e cheias, recorrentes no centro e sul do país.

No seio das comunidades rurais de Moçambique, as mulheres encontram-se numa situação de particular desvantagem, principalmente no que se refere ao acesso à educação e saúde. A maioria das mulheres destas comunidades trabalha na agricultura, sendo responsáveis principalmente 
pelas colheitas onde a carga de trabalho é pesada e importante no rendimento familiar, pois está dependente da produtividade do trabalhador. No entanto têm pouco acesso ou controlo sobre os recursos de produção (IFAD, 2011).

No caso de Cabo Verde, o ensino superior desenvolveu-se mais recentemente do que em Moçambique. Até à criação da Universidade Jean Piaget (instituto privado) em 2001, o país apenas contava com três instituições públicas de pequena dimensão (Instituto Superior de Educação - ISE; Instituto Superior de Engenharia e Ciências do Mar - ICSEMAR e o Instituto Nacional de Gestão e Administração - INAG). Em 2006 os três institutos acabaram por se associar, criando a Universidade de Cabo Verde, sendo a primeira e única universidade pública no país (o processo só se concluiu em 2008 com a integração absoluta dos três institutos).

A agricultura em Cabo Verde é limitada pela disparidade das secas severas e inundações recorrentes que afetam o arquipélago. Com apenas $10 \%$ de solo arável, escassez de recursos minerais, clima árido e terreno montanhoso, a produção agrícola é extremamente limitada, resultando em colheitas irregulares e períodos de fome. Cerca de 90\% dos alimentos são importados. Entre as principais culturas de consumo local destacam-se o milho, cana-de-açúcar, fava, batata e amendoim.

O rápido crescimento económico desde o início da década de 1990 reduziu a pobreza em Cabo Verde. De acordo com o Banco Mundial, a percentagem de população na situação de pobreza absoluta reduziu de 49\% em 1988-89 para 37\% em 2001-02, 27\% em 2007 e 24\% em 2010. No entanto a desigualdade permanece elevada, tendo aumentado durante a década de 1990 e permanecido elevada desde então, com um coeficiente de Gini para o consumo de 0,49 (2007). A redução de pobreza tem-se revelado mais lenta em áreas rurais, onde residem $72 \%$ dos pobres dos quais $30 \%$ em estado de pobreza absoluta, contrastando com os $12 \%$ registados entre a população urbana. Os valores variam de acordo com a ilha. As ilhas com maior população rural (Santo Antão, Santiago, São Nicolau e Fogo) apresentam os maiores índices de pobreza e escassez de alimentos.

Neste contexto, tem havido um crescente reconhecimento da necessidade de desenvolver novos programas educacionais nos países em 
desenvolvimento que reflitam as mudanças e dinâmicas em curso no que concerne a novos conceitos de culturas e gestão territorial, contribuindo deste modo para responder as crescentes necessidades de profissionais qualificados em agricultura.

De modo a responder às evoluções e alterações das necessidades da agroindústria e dos próprios produtores, os programas devem ter em consideração uma profunda compreensão dos condicionalismos na implementação da agricultura de precisão em países em desenvolvimento e a integração de abordagens revolucionárias na gestão de informação agrícola e no desenvolvimento de técnicas adequadas para os locais-alvo, recursos e características dos produtores (agricultura familiar e de subsistência).

\section{O Projeto Agim}

Com base na experiência do Instituto de Superior de Estatística e Gestão de Informação - ISEGI-NOVA em redes de cooperação no campo da Ciência e Tecnologias de Informação Geográfica (PAINHO, 1995 e 1999; PAINHO et al, 2007) e de modo a dar uma reposta adequada aos problemas acima descritos relativos a Moçambique e Cabo Verde, o ISEGI- NOVA (como instituição requerente) em parceria com a Escola Superior de Ciências Agrárias e Ambientais da Universidade de Cabo Verde (UniCV) e a Faculdade de Ciências Agrárias da Universidade Católica de Moçambique (UCM) e com o apoio da Esri Portugal (membro associado), submeteu uma proposta ao Programa EDULINK ${ }^{1}$, o projeto AgIM (Agricultural Information Management and Precision Farming).

O projeto tem uma duração esperada de 42 meses e pretende atingir o reforço institucional e humano das duas IES parceiras, bem como o apoio no desenvolvimento de um curso de pós-graduação e mestrado

1 O programa EDULINK é financiado pela União Europeia (UE) e implementado pelo Secretariado ACP (African, Caribbean and Pacific Group of States), que tem o objetivo de melhorar a eficácia e o impacto da cooperação UE-ACP no ensino superior. Esta parceira promove a integração regional no domínio do ensino superior através de redes institucionais e o apoio a um sistema de ensino de qualidade, que consiga responder às necessidades do mercado de trabalho e de acordo com as prioridades de desenvolvimento económico dos países ACP (Secretariado ACP, 2007,2008a,2008b). Para mais informações consultar: http://www.acp-edulink.eu 
de referência em boas-práticas de gestão de informação agrícola e agricultura de precisão em países em vias de desenvolvimento, que irá cobrir três áreas fundamentais:

1. Agricultura de precisão: Adequação dos currículos para a realidade dos países em desenvolvimento (comunidades rurais dedicadas essencialmente à agricultura de subsistência) e através da combinação de integração de abordagens inovadoras na gestão do território com práticas e conhecimentos locais;

2. Sistemas de monitorização e agricultura sustentável: Os conceitos de agricultura de precisão e sustentabilidade são praticamente indissociáveis, devendo ser vistos como fundamentais em estratégias de promoção de desenvolvimento agrícola principalmente no que concerne a países em desenvolvimento;

3. Desenvolvimento da cadeia de produção agrícola: Num período de tempo mais alargado, espera-se que o projeto contribua para uma gestão mais eficiente da produção e rendimentos dos agricultores locais, para além de melhorias no acesso aos mercados agrícolas.

O objetivo específico do projeto passa por melhorar a gestão académica, administrativa e tenológica das IES parceiras, de modo a sustentar a implementação e transferência de duas edições do curso de pós-graduação e mestrado. O currículo tem como base boas-práticas em gestão de informação agrícola e agricultura de precisão, tendo em consideração os seus últimos desenvolvimentos e condições específicas para a sua introdução em países em vias de desenvolvimento. Existe também o objetivo de estabelecer um enquadramento comum de garantia de qualidade relacionada com o reconhecimento/acreditação do programa de pós-graduação e mestrado AgIM pelas autoridades competentes.

Está prevista a organização de duas edições do AgIM em Moçambique e Cabo Verde e o estabelecimento de dois projetos-piloto de demonstração (um em cada país), que irão desempenhar um papel crucial na implementação do conhecimento adquirido ao longo das unidades curriculares. Para facilitar a transferência de conhecimento será desenvolvido um repositório de práticas e trabalho de campo que servirá como suporte na adaptação 
e atualização dos métodos e técnicas adotados.

A primeira edição do AgIM será coordenada e conduzida por um corpo docente do ISEGI-NOVA juntamente com dois professores da UCM e UniCV com formação em tecnologias SIG. Na segunda edição os docentes locais irão estar envolvidos diretamente em todas as atividades, passando os docentes do ISEGI a supervisionar as aulas (à distância e, quando necessário, no local).

\section{Metodologia Apresentada}

O projeto pretende prestar particular atenção às carências das duas instituições parceiras ao nível administrativo, académico e tecnológico (para além das questões relacionadas com a agricultura de precisão). O projeto AgIM irá implementar um conjunto de atividades práticas e em dois projetos-piloto de demonstração, dando oportunidade aos estudantes de aprender métodos de integração de agricultura de precisão e de processos de tomada de decisão. Deste modo é esperado que os alunos atinjam com sucesso os passos fundamentais na adoção e aplicação da agricultura de precisão (KITCHEN et al, 2002):

1. Compreensão do conceito de dados espaciais e sua gestão e importância;

2. Aprendizagem do uso de tecnologias (GPS, deteção remota, etc.) que contribuem para a recolha de dados intensiva de informação qualidade a custo reduzido.

3. Aprendizagem de software SIG (Sistemas de informação geográfica).

4. Compreensão dos fatores que influenciam a extensão, estabilidade e rendimento da atividade agrícola.

5. Realização de trabalho de campo de modo a compreender as causas da variabilidade (integração de conhecimento local com conhecimento técnico especializado).

6. Otimização da estão estratégica por meio de amostragem e ensaios no local (explorações agrícolas).

Através destes passos, os estudantes irão adquirir conhecimento das várias facetas da agricultura de precisão. Após a formação do curso AgIM, estarão preparados para desempenhar um papel importante como especialistas qualificados no sector agrícola, podendo desenvolver as 
seguintes tarefas:

- Aquisição de informação relacionada com solos e dados de produção e sua integração em aplicações de Sistemas de informação geográfica.

- Documentação e tratamento de dados relativos a agricultura de precisão.

- Classificação de camps agrícolas em zonas georreferenciadas tendo como base características de solo e potencial de produção.

- Avaliação de tipo de cultivo adequados a áreas especificas, tendo como base a analise de dados geoespaciais.

A metodologia proposta assenta num conjunto de objetivos:

- Melhorar a gestão administrativa, académica e tecnológica das duas instituições parceiras de modo a apoiar a implementação de duas edições do curso de pós- graduação e mestrado AgIM.

- Desenvolver o curso AgIM tendo em consideração os últimos avanços no campo da agricultura de precisão e condições para a sua implementação em países em vias de desenvolvimento.

- Estabelecer um quadro comum de garantia de qualidade.

- Promover o reconhecimento/acreditação do curso AgIM pelas autoridades competentes.

- Criação de uma rede interinstitucional de modo a promover a investigação, pesquisa e inovação tecnológica entre os parceiros.

- Promover o envolvimento ativo dos beneficiários e a relevância do AgIM no contexto local e regional.

- Garantir a implementação e execução do programa de acordo com as necessidades locais e regionais do mercado trabalho e em conformidade com as condições socio económicas e prioridades de desenvolvimento de cada país.

- Integrar um conjunto de soluções de modo a tornar o AgIM autossustentável com especial atenção para a mitigação do problema da fuga de cérebros dos dois países.

Foi definido um conjunto de grupos-alvo que irão beneficiar diretamente com a implementação do curso de pós-graduação e mestrado AgIM:

- Estudantes - São esperados 70 estudantes nas duas primeiras edições, 
dividindo- se em 40 em Moçambique e 30 em Cabo Verde. Será realizado um esforço adicional (contactos com instituições de igualdade locais e atribuição de um ponto extra a candidatas ao AgIM) de modo a que pelo menos $30 \%$ dos alunos sejam do sexo feminino de modo a aumentar o número de mulheres qualificadas no sector agrícola.

- Docentes - Formação de 16 professores no ramo da agricultura e tecnologias de informação geográfica: 8 docentes provenientes da UCM e 8 d UniCV.

- Gestão/administração - Através de cursos de formação organizados paralelamente ao AgIM, espera-se a qualificação de staff administrativo, financeiro e relações externas. No total prevê-se a formação de 4 elementos de cada instituição parceira.

- Técnicos de Tecnologias de Informação e Comunicação (TIC) Formação de 2 técnicos TIC, que serão responsáveis por desenvolver os websites locais e apoio tecnológico na utilização da plataforma e-learning e seus conteúdos.

- Pequenos agricultores/comunidades locais - Pequenos agricultores e comunidades diretamente envolvidos nos dois projetos-piloto de demonstração (1 em cada país).

A médio/longo termo os beneficiários finais do projeto serão todos os cidadãos, agricultores, comunidades, instituições e organizações em Cabo Verde e Moçambique que irão beneficiar por um aumento do numero de profissionais qualificados em agricultura no sector da alimentação, com uma melhor compreensão das necessidades da produção e produtores à escala local e com conhecimento para facilitar os processos de tomada de decisão e aumento dos rendimentos provenientes da atividade. Ao responder diretamente aos problemas detetados no sector agrícola, contribui-se para uma estratégia de redução de pobreza, aumento do fornecimento de alimentação e implementação de boas-práticas e gestão de recursos naturais. Especificamente os principais beneficiários da ação serão:

- Comunidades locais 
- Consumidores

- Mulheres

- Pessoas afetadas com VIH/SIDA

- Organizações comerciais/agronegócio

- Outras organizações educacionais

- Instituições de investigação/investigadores

- Outros estudantes

- Autoridades locais

- Administração pública

- Organizações não-governamentai

A implementação do projeto compreende ainda um conjunto de atividades divididas em seis áreas específicas:

1. Gestão das atividades da ação: Transversal a todo o projeto. Reúne os meios para a execução do projeto, implementação (incluindo monitorização, revisão e elaboração de relatórios), avaliação e auditoria.

2. Criação de capacidades académicas e gestão dos parceiros: $O$ objetivo desta atividade é a melhoria da capacidade académica e de gestão/ administração geral dos parceiros. Esta atividade será composta por um conjunto de cursos de formação especialmente concebidos para melhorar as qualificações dos docentes e de gestão/administração dos dois parceiros. Estão previstos 6 cursos:

- Problem based-learning: Formação dirigida a docentes. Sessões orientadas para o desenvolvimento de projetos.

- Teorias de desenvolvimento curricular: Formação dirigida a docentes. Estudo aprofundado dos fundamentos, teorias e procedimentos de desenvolvimento curricular e avaliação.

- Tecnologias educacionais: Formação dirigida a docentes e staff TIC. Sessões especialmente concebidas para melhorar e facilitar o processo de aprendizagem através do uso de ferramentas de e-learning relevantes e inovadoras e gestão de infraestruturas tecnológicas adequadas. 
- Atual estado da arte da agricultura de precisão: Formação dirigida a docentes e staff TIC. Estado da arte das abordagens e métodos utilizados na

- agricultura de precisão, através da utilização de materiais e conteúdos curriculares relevantes e inovadores.

- Gestão de projetos internacionais: Formação dirigida a staff administrativo e docentes interessados em submeter projetos internacionais. Direcionado principalmente para recursos humanos das instituições parceiras, mas

- também estará disponível para outros interessados como instituições governamentais, organizações não-governamentais (ONG's) e consultores. O curso irá focar-se em padrões, ferramentas e diretrizes de gestão de projetos estabelecidos para apoiar boas-práticas de gestão e tomadas de decisão, ao longo de um ciclo de gestão de um projeto.

- Gestão de relações externas no ensino superior: Formação dirigida a staff administrativo e docentes interessados em submeter projetos internacionais. As atividades de formação passarão por temáticas como estágios e integração dos estudantes no mercado de trabalho, colaborações externas, criação de redes institucionais com os principais parceiros nacionais/ internacionais, promoção do envolvimento com as comunidades locais, ONG's e autoridades locais.

3. Desenvolvimento do currículo AgIM: Este bloco compreende um conjunto de atividades relacionadas com a construção do currículo do curso AgIM, lidando com todos os aspetos da criação, estruturação e organização do currículo, incluindo todas as atividades relacionadas com a criação e produção dos seus elementos- chave (e-books, exames de avaliação, bibliografia recomendada, recursos de internet, exercícios práticos, estudos de caso, apresentações de palestras, atividades de aprendizagem, repositórios de dados, etc.).

\section{Criação e gestão de dois projetos-piloto de demonstração: Este} bloco inclui uma vasta gama de atividades relacionadas com a criação de dois projetos-piloto de demonstração (um em cada país parceiro) que irão desempenhar um papel crucial em toda a ação. Espera-se que o conhecimento e a experiência adquirida através da implementação destes 
dois projeto-piloto irão contribuir para desenvolver uma análise crítica e avaliação da adequação do curso de mestrado, fornecendo simultaneamente uma perceção mais profunda sobre as abordagens e estratégias que podem apoiar programas de educação mais eficazes e relevantes em Moçambique e Cabo Verde.

5. Duas edições do curso de pós-graduação e mestrado AgIM: Este bloco inclui as atividades de ensino (palestras, sessões tutoriais, seminários, projetos de supervisão/dissertação, avaliação, etc.), relacionados com as duas edições do Programa de Pós-Graduação e Mestrado AgIM em Moçambique e Cabo Verde.

6. Desenvolvimento de ações de promoção e divulgação: Esta atividade compreende um conjunto de ações de divulgação e exploração projetados para integrar e manter boas-práticas de educação em agricultura de precisão em países em desenvolvimento e para incrementar as redes interinstitucionais entre os parceiros.

\section{Modelo pedagógico}

O Programa de Pós-Graduação e Mestrado do curso AgIM tem a duração de três semestres e integra um conjunto de unidades curriculares de natureza obrigatória. O primeiro semestre do curso consiste em quatro unidades curriculares que proporcionam competências e conhecimentos específicos considerados essenciais para atingir os objetivos definidos para o programa de estudos:

- SIG e Agricultura de Precisão

- Métodos de Pesquisa

- Aquisição de Dados

- Deteção Remota e Análise Espacial

- O segundo semestre do curso apresenta um conjunto de quatro unidades curriculares com um maior nível de especialização e aprofundamento:

- Métodos Quantitativos

- Agricultura de Precisão

- Gestão de Informação Agrícola

- Implementação da Agricultura da Precisão na Comunidade 
Os objetivos e o conteúdo das unidades visam a aquisição de competências necessárias para a continuação do processo de aprendizagem e conhecimento, promovendo a investigação científica e utilização de técnicas e abordagens adequadas para a resolução de problemas complexos na área de Gestão de Informação Agrícola e Agricultura de Precisão. O terceiro semestre do curso é dedicado à preparação da dissertação.

A obtenção de um diploma de pós-graduação é dependente da realização dos créditos associados às oito unidades curriculares que integram o $1^{\circ}$ e o $2^{\circ}$ semestre. A concessão do grau de mestre exige, além da aprovação na componente curricular do $1^{\circ}$ e do $2^{\circ}$ semestre, a elaboração de uma dissertação, sua discussão e aprovação.

As unidades curriculares serão lecionadas de forma sequencial. O início de uma unidade curricular coincide necessariamente com o término da unidade curricular que lhe antecede. Cada unidade curricular tem uma duração prevista de 5 semanas, conforme esquema que se segue (ver figura 1):

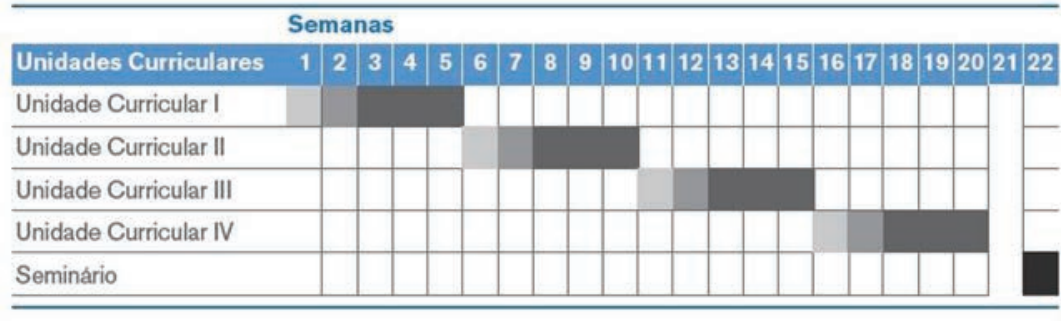

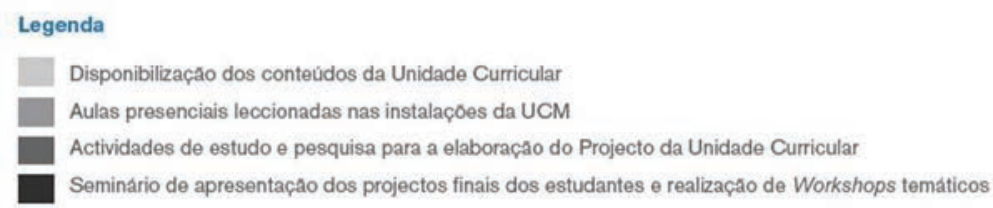

Figura 1 - Sequência das Unidades Curriculares

No final de cada semestre irão decorrer as defesas dos trabalhos realizados pelos alunos (um projeto por unidade curricular). As discussões estão abertas, podendo assistir, para além de docentes, alunos e staff das IES parceiras, elementos de ONG's, cidadãos, produtores locais, entre outros. 


\section{SituAÇão ATUAl do PROJeto Agim}

O início formal do projeto decorreu no First Steering Committee (27 e 28 de Novembro de 2013 - ISEGI-NOVA) onde se reuniram todos os parceiros do consórcio: ISEGI-NOVA, UniCV, UCM e Esri Portugal (ver figura 2). Esta reunião teve como objetivo a apresentação dos objetivos e sua discussão, calendarização das aulas e datas importantes no projeto e distribuição de tarefas entre os parceiros.

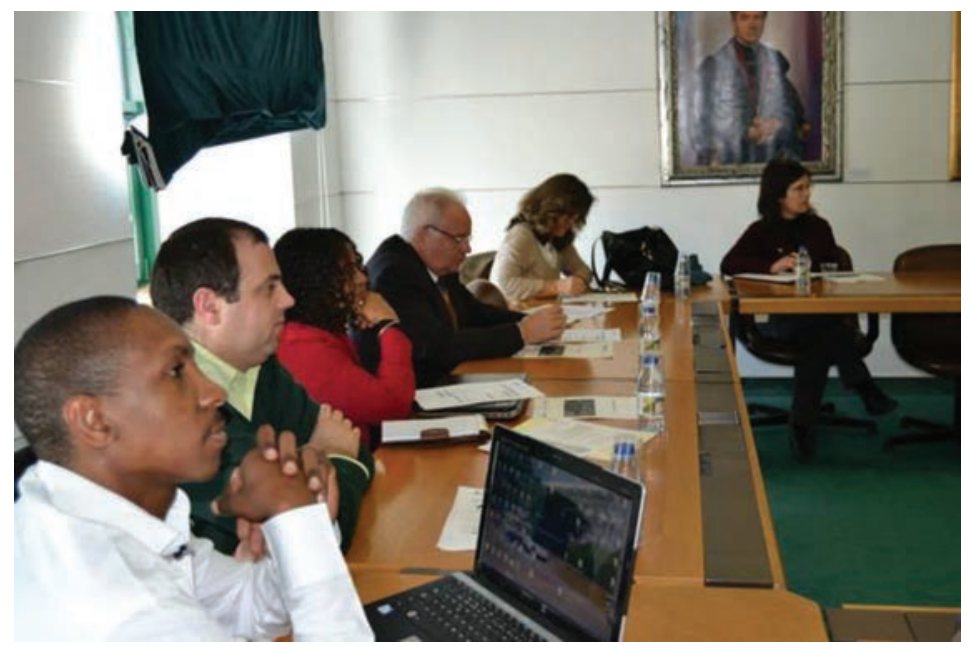

Figura 2 - Sequência das Unidades Curriculares

Para efeitos de informação e divulgação, foi criado um website oficial do projeto (http://agim.isegi.unl.pt), sendo possível consultar os objetivos do AgIM, participantes, unidades curriculares e cursos de formação a serem lecionados durante o período de implementação do projeto (ver figura 3).

Cada parceiro conta também com um website próprio integrado no principal, onde se encontra reunida a informação específica de cada IES (http://agim.isegi.unl.pt/pt/ucm/ - UCM; http://agim.isegi.unl.pt/pt/unicv/ - UniCV) (ver Figuras 4 e 5). 


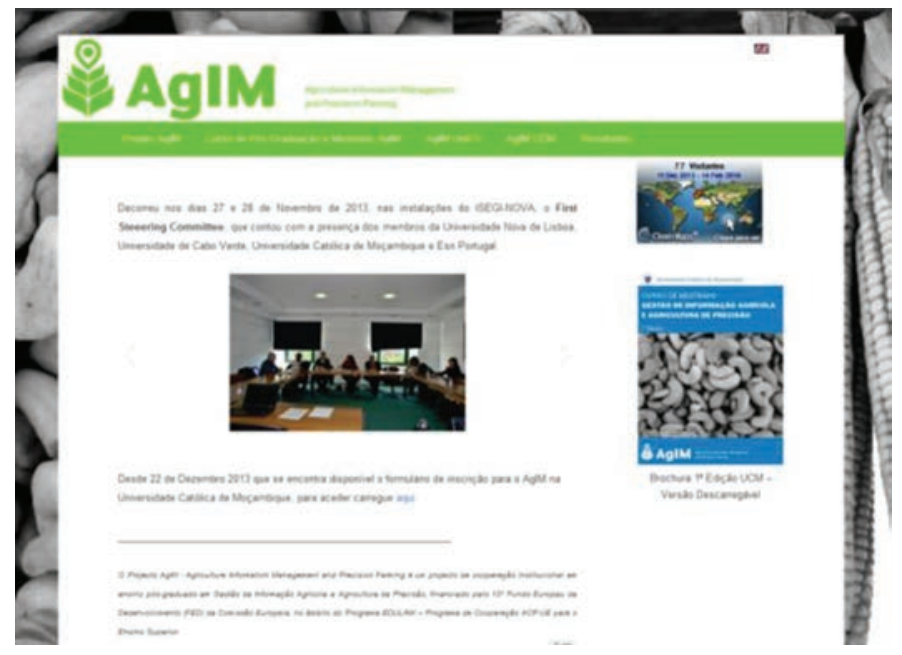

Figura 3 - Página principal do website AgIM

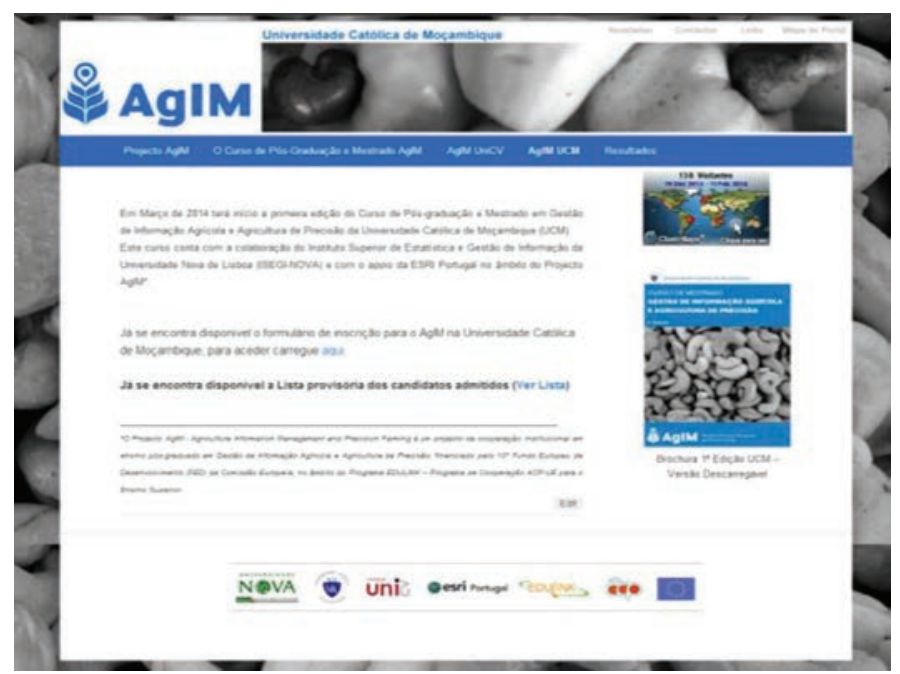

Figura 4 - Página principal do website AgIM - UCM 


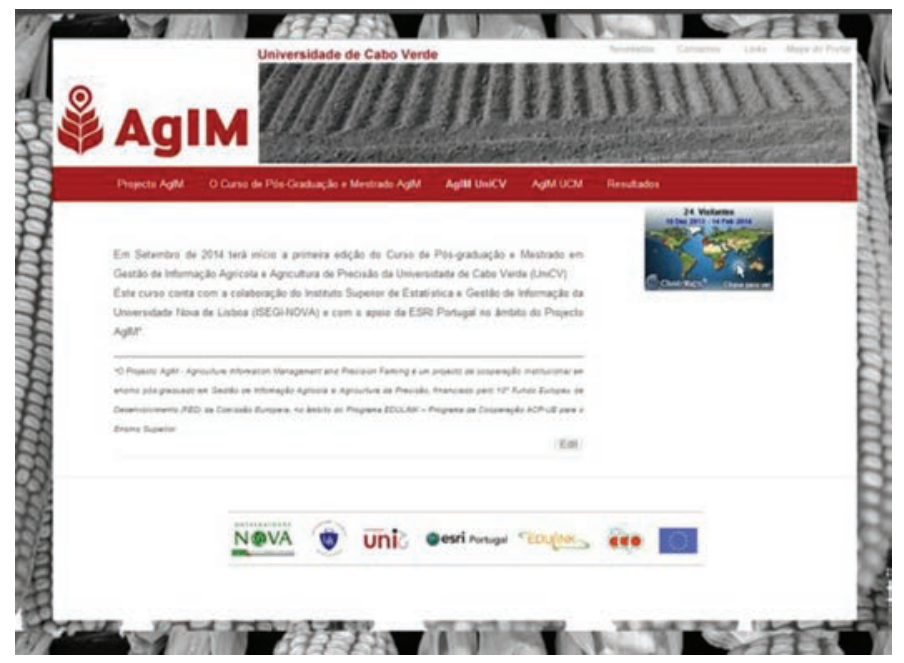

Figura 5 - Página principal do website AgIM - UniCV

O curso AgIM será lecionado em regime de blended learning ( $b$ -learning), ou seja reúne as características referentes ao e-learning (disponibilização dos conteúdos online e apoio dos docentes à distância) com aulas presenciais. A componente do e-learning é garantida por meio de uma plataforma Moodle desenvolvida especialmente para o AgIM (http://moodle.agim.isegi.unl.pt/). Os alunos do curso terão acesso ao Moodle AgIM onde estão disponibilizados os conteúdos das unidades curriculares para além de elementos de avaliação. A plataforma servirá também como um ponto de contacto entre os docentes e estudantes (ver figura 5).

A $1^{\text {a }}$ edição do AgIM irá decorrer numa primeira fase em Moçambique, a partir de Março de 2014. Até ao momento contam-se 17 candidatos, a maioria dos quais com formação académica em ciências agrárias, como seria de esperar de um curso de agricultura de precisão, os restantes são originários das áreas de gestão, ciências biológicas e química marinha. 


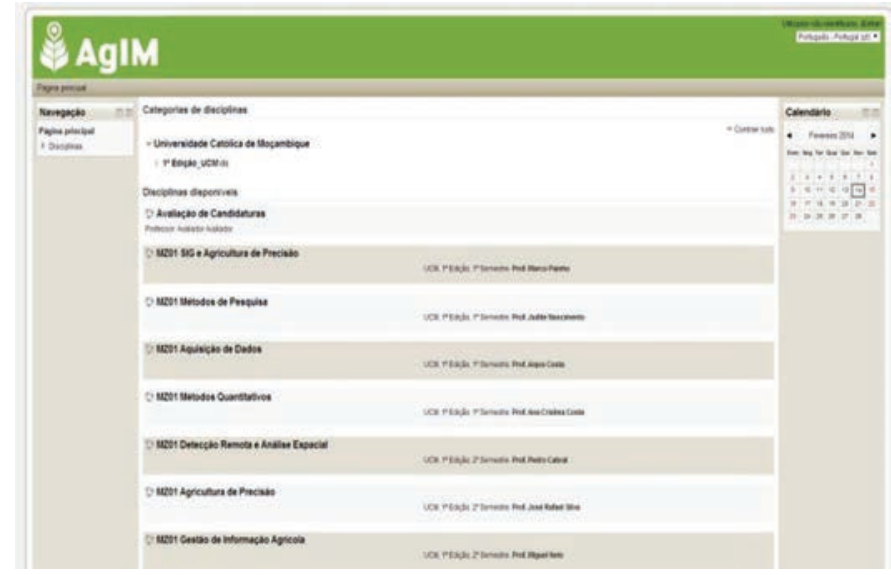

Figura 6 - Página principal do Moodle AgIM

\section{CONCLUSÃo E RESULTADOS ESPERADOS}

Através dos objetivos propostos e metodologia adotada, foi delineado um conjunto de resultados esperados. Em primeiro lugar e ligado diretamente ao curso de pós-graduação e mestrado AgIM prevê-se a formação de 16 professores ( 8 em cada instituição parceira), que serão responsáveis por lecionar as unidades curriculares na $2^{\text {a }}$ edição do curso, com o apoio de docentes do ISEGI-NOVA, permitindo deste modo a sustentabilidade da ação. Este conjunto de docentes será selecionado dos alunos que irão frequentar a $1^{a}$ edição. Com a organização de uma série de cursos de formação, pretende-se a qualificação de pessoal administrativo, gestão e de tecnologias de informação ( 5 em cada IES) o que irá permitir colmatar carências detetadas e valorizar as instituições parceiras.

Outro grande objetivo passa pela organização de dois projetos-piloto de demonstração em agricultura de precisão (Cabo Verde e Moçambique). Estes projetos além de terem a função de possibilitar aos alunos uma experiência in situ da aplicação dos conhecimentos adquiridos ao longo das unidades curriculares, permite também o contacto com as comunidades e produtores locais, dando a conhecer os seus problemas e facilitando a adaptação das técnicas de agricultura de precisão em países de desenvolvimento (ver Figuras 7 e 8). 


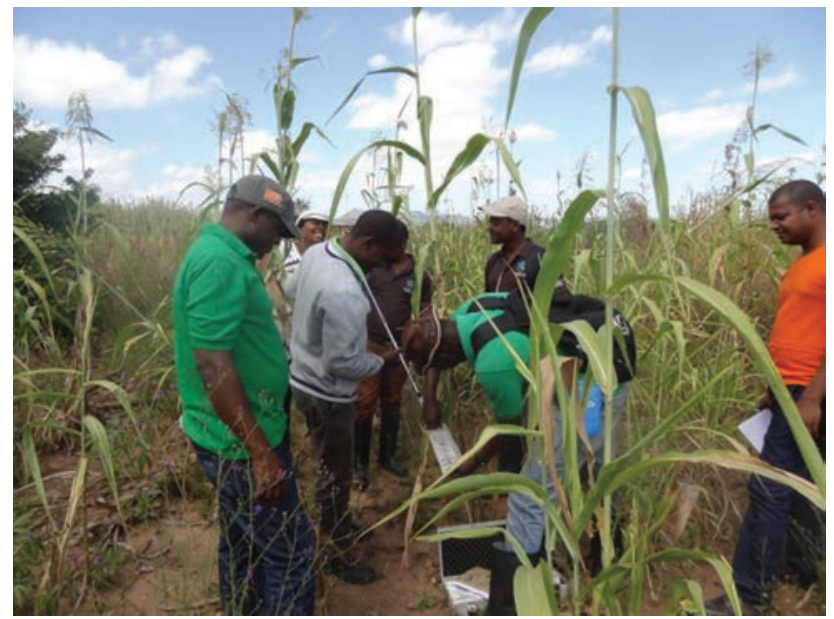

Figura 7 - Alunos do AgIM em trabalho de campo (Moçambique)

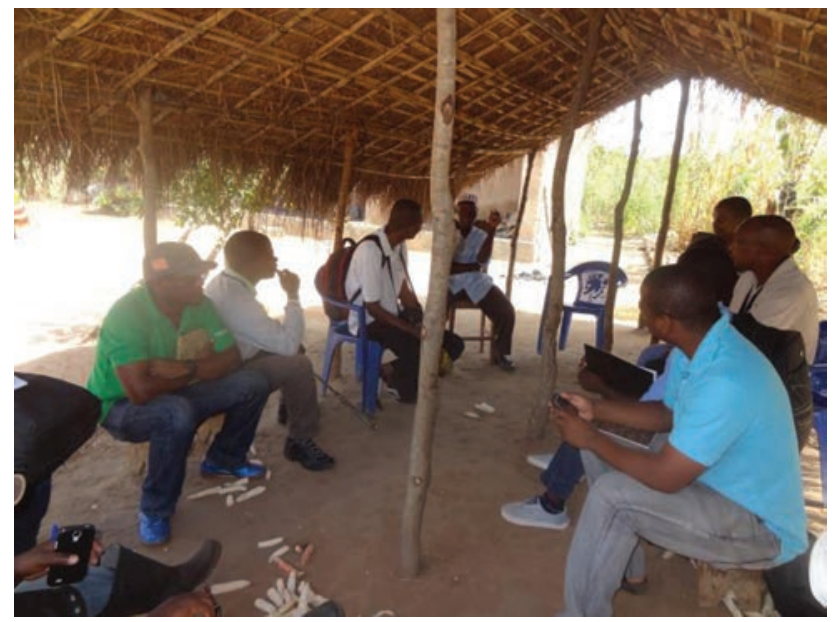

Figura 8 - Alunos do AgIM em trabalho de campo - contato com as comunidades locais (Moçambique)

Os projetos piloto servirão de base para a criação de uma plataforma de boas-práticas de agricultura de precisão em países em desenvolvimento (estando também previsto um livro sobre o tema) e também um pequeno documentário de 15 minutos relativo a estas ações. Para efeitos de divulgação da temática da agricultura de precisão, serão organizadas duas conferências em cada IES.

O AgIM será pioneiro no âmbito do ensino pós graduado em agricul- 
tura de precisão nos dois países envolvidos no processo. Para além da formação de técnicos especializados em um sector determinante para o desenvolvimento socioeconómico de Cabo Verde e Moçambique, permite também adaptar e testar as tecnologias da agricultura de precisão em pequenas comunidades rurais que praticam essencialmente a atividade como meio de subsistência.

O projeto aborda também com especial atenção as componentes de gestão/administração, académica, investigação e tecnológica das IES parceiras, levando a que exista um esforço, por meio da organização de cursos/workshops para responder aos problemas encontrados de modo a valorizar as instituições e o seu staff. Com o reforço das relações externas dos parceiros juntamente com o conhecimento técnico adquirido, tanto a UniCV como a UCM poderão tornar-se centros de referência no âmbito da gestão de informação agrícola e agricultura de precisão, e sua aplicação em países em desenvolvimento.

\section{BIBLIOGRAFIA}

ADHIKARI, Kabindra et al. (2009) "Site specific land management: General concepts and applications”. In JRC Scientific and Technical Reports, Luxemburgo. Disponível online no endereço url:http://eusoils.jrc.ec.europa.eu/esdb_archive/eusoils_docs/other/EUR23978.pdf (acedido em 17 Fevereiro, 2014).

COOK, Simon et al. (2003) - "Precision agriculture" in Stafford, J.V \& Werner, A (eds.): Wageningen Academic Publishers, Wageningen, 115-119.

INTERNATIONAL FUND FOR AGRICULTURAL DEVELOPMENT (2011) - "Republic of Mozambique: Country strategic opportunities programme”. IFAD, Roma, Itália. Disponivel online no endereço url: http://www.ifad.org/gbdocs/ eb/103/e/EB-2011-103-R-13.pdf (acedido em 13 Fevereiro, 2014).

KITCHEN, Newell et al. (2002) - "Educational needs of precision agriculture" Precision Agriculture, $N^{\circ} 3,341-351$.

LUMPKIN, Thomas (2011) - "Help for developing countries in a world of rising grain prices"

ISHS Acta Horticulturae, Vol. 916, 31-46.

MAOHUA, Wang (2001) - "Possible adoption of precision agriculture for developing countries at the threshold of the new millennium" Computers and electronics in agriculture, Vol. 30, $\mathrm{N}^{\circ} 3,45-50$. 
MINISTÉRIO DO ENSINO SUPERIOR, CIÊNCIA E TECNOLOGIA (2000) - "Strategic plan of higher education in Mozambique". Ministério do Ensino Superior, Ciência e Tecnologia, Maputo, Moçambique. Disponível online no endereço url: http://planipolis.iiep.unesco.org/upload/Mozambique/Mozambique_ HE_Strategic_Plan\% 202000_2010.pdf (acedido em 13 Fevereiro, 2014).

MISHRA, Ashish et al. (2003) - "Operationalization of precision farming in India". In Map India 2003. Disponível online no endereço url: http://gisdevelopment.net/application/agriculture/overview/pdf/127.pdf (acedido em 17 Fevereiro, 2014).

MONDAL, Pinaki \& BASU, Manisha (2009) "Adoption of precision agriculture in India and in some developing countries: scope, present, status and strategies" Progress in natural science, Vol. 19, N6, 659-666.

PAINHO, Marco (1995) “Teaching GIS to multiple background students: ISEGI's experience" Proceedings of the Joint European Conference and Exhibition on Geographical Information - from Research to Application through Cooperation, Vols. 1 e 2, 281-285.

PAINHO, Marco (1999) - Teaching GIS in Information Management - A discussion of teaching methods, options and curricula for GIS teaching in higher education. Instituto Superior de Estatística e Gestão de Informação da Universidade Nova de Lisboa, Lisboa.

PAINHO, Marco et al (2007) - "eduGI Project - An International Network for GIScience Courses Exchange”. In Proceedings of the 7th Annual ESRI Education User Conference, San Diego, Estados Unidos. Disponível online no endereço url:http://proceedings.esri.com/library/userconf/educ07/educ/ papers/pap_1499.pdf (acedido em 13 Fevereiro, 2014)

RILWANI, Momoh \& IKHUORIA, Isi (2006) - "Precision farming with geoinformatics: a new paradigm for agricultural production in a developing country" Transactions in GIS, Vol. 10, $\mathrm{N}^{\circ} 2,177-197$.

WIEBOLD, William et al. (1998) - "Determining barriers to adoption and research needs of precision agriculture". In Report to the North Central Soybean Research Program, Missouri, Estados Unidos. Disponível online no endereço url: http://www.fse.missouri.edu/mpac/pubs/parpt.pdf (acedido em 15 Janeiro, 2014) 
Série Documentos

Imprensa da Universidade de Coimbra

Coimbra University Press

2015

- U M

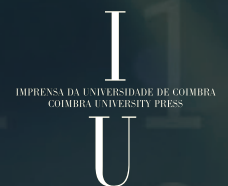

